\title{
EFFICIENT, ADAPTIVE ENERGY STABLE SCHEMES FOR THE INCOMPRESSIBLE CAHN-HILLIARD NAVIER-STOKES PHASE-FIELD MODELS
}

\author{
YING CHEN ${ }^{\natural}$ AND JIE SHEN ${ }^{\dagger}$
}

\begin{abstract}
In this paper we develop a fully adaptive energy stable scheme for Cahn-Hilliard NavierStokes system, which is a phase-field model for two-phase incompressible flows, consisting a CahnHilliard-type diffusion equation and a Navier-Stokes equation. This scheme, which is decoupled and unconditionally energy stable based on stabilization, involves adaptive mesh, adaptive time and a nonlinear multigrid finite difference method. Numerical experiments are carried out to validate the scheme for problems with matched density and non-matched density, and also demonstrate that CPU time can be significantly reduced with our adaptive approach.
\end{abstract}

\section{INTRODUCTION}

In recent years the phase field methods, also called diffuse interface methods, have been successfully used to study a variaty of interfacial phenomena (cf. [12, 3, 21, 15, 18, 36, 19], the recent reviews $[25,16]$ and the references therein). The basic idea for the phase field methods can be traced back to [24] and [30], which was originally developed to model solid-liquid phase transitions, where the interface is represented as a thin transition layer between two phases which is indicated by an auxiliary function. A particular advantage of the phase field methods is that the system can be derived from an energy variational approach, which leads to well-posed nonlinearly coupled systems that satisfy thermodynamically consistent energy dissipation laws. That makes it possible to design an numerical scheme that preserves the energy law at the discrete level (cf., for instance, $[11,17,4])$ in order to ensure the stability of the numerical scheme and the accuracy of the solution.

The challenges and complexities of problems involving the mixtures of different materials lie in their multiscale and sometimes multiphysics nature. It is the competitions and couplings between these effects which determine the overall properties of the mixtures. A main difficulty in dealing with non-matching densities can be seen in the basic macroscopic (continuum) mass conservation $\rho_{t}+\operatorname{div}(\rho u)=0$ and (macroscopic) incompressibility $\operatorname{div} u=0$. As the density $\rho$ is an macroscopic quantity, it may be different with the direct average from microscopic descriptions, such as from the phase fields. For instance, the mixtures of two incompressible fluids may not be incompressible, for we have to take into consideration of the interaction between two different particles. Various approaches have been proposed in the literature. Traditionally, they can be classified into two categories: incompressible or quasi-incompressible. In the former approach, the volume averaged velocity is assumed to be incompressible everywhere, including the interfacial region. In the latter approach, on the other hand, the mass averaged velocity is assumed to satisfy the mass conservation instead of incompressibility, leading to a slightly compressible mixture inside the interfacial region. In [21], the authors derived a quasi-incompressible phase model which allows the mixture to be slightly compressible inside the interface, see also [1]; a similar quasi-incompressible model, which

Key words and phrases. Phase-field, two-phase flow, Navier-Stokes, variable density, adaptivity, stability, energy stable schemes.

$\curvearrowleft$ Department of Mathematics, Purdue University, West Lafayette, IN 47907 (chen1447@purdue.edu).

$\dagger$ Department of Mathematics, Purdue University, West Lafayette, IN 47907 (shen@math.purdue.edu).

1

(C) 2015. This manuscript version is made available under the Elsevier user license

http:/www.elsevier.com/open-access/userlicense/1.0/ 
admits an energy law, was proposed recently in [26]. On the other hand, incompressible phase-field models were derived in $[5,10,27,2]$. In particular, the model in [2] is thermodynamically consistent, frame invariant and admits an energy law, and in [28], the authors developed a semi-discrete-in-time scheme for this model which is totally decoupled, linear and unconditionally energy stable.

It is well-known that the accuracy of the phase-field models depends on the the interfacial width which has to resolved by the spatial discretization. Since the interfacial region usually occupies a small portion of the domain, it is very costly to resolve the interface with a uniform grid. On the other hand, the solution variation can be very different at different times so it is also very beneficial to have an adaptive procedure to select time steps automatically. The main purpose of this paper is to develop a fully discretized and fully adaptive numerical scheme for the model in [2] based on the semi-discrete-in-time scheme in [28] and a finite difference multigrid method in space.

The paper is organized as follows. In Section 2, we describe Cahn-Hilliard Navier-Stokes models of two-phase incompressible flows and present decoupled, energy stable schemes for both uniform constant density and variable density cases. In Section 3, we construct a fully discrete energy stable scheme for both uniform constant density and variable density cases. Adaptive mesh and time adaptive strategies are also introduced. Numerical simulations and some concluding remarks are presented in Section 4.

\section{CAHN-HILliard PHASE-FIEld MODElS}

In this section, we recall the Cahn-Hilliard Navier-Stokes phase-field models and their time discretizations for a mixture of two immiscible, incompressible fluids in a confined domain $\Omega \subset \mathbf{R}^{d}$, $d=2$ or 3 , with densities $\rho_{1}, \rho_{2}$ and viscosities $\nu_{1}, \nu_{2}$, respectively. We first introduce a phase function (macroscopic labeling function) $\phi$ such that

$$
\phi(\mathbf{x}, t)= \begin{cases}1 & \text { fluid } 1, \\ -1 & \text { fluid 2 }\end{cases}
$$

with a thin, smooth transitional layer of width $O(\eta)$. The (equilibrium) configurations and patterns of this mixing layer, in the neighborhood of the level set $\Gamma_{t}=\{\mathbf{x}: \phi(\mathbf{x}, t)=0\}$, is determined by the microscopic interactions between fluid molecules. For the isotropic interactions, the classical self consistent mean field theory (SCMFT) in statistical physics [7] gives the following Ginzburg-Landau type of Helmholtz free energy functional:

$$
E(\phi, \nabla \phi)=\int_{\Omega} \lambda\left(\frac{1}{2}|\nabla \phi|^{2}+F(\phi)\right) d x
$$

where the first term represents the hydro-philic type (tendency of mixing) of interactions between the materials and the second term, the double well bulk energy $F(\phi)=\frac{1}{4 \eta^{2}}\left(\phi^{2}-1\right)^{2}$, implies the hydro-phobic type (tendency of separation) of interactions. As the consequence of the competition between the two types of interactions, the equilibrium configuration will include a diffusive interface with thickness proportional to the parameter $\eta$ (cf., for instance, [36]); and, as $\eta$ approaches zero, we expect to recover the sharp interface separating the two different fluids.

2.1. Case of matched density. We first consider the mixture of two incompressible fluids with constant, uniform density, i.e., $\rho_{1}=\rho_{2}$. The dynamics of the phase function $\phi$ is governed by the Cahn-Hilliard gradient flow (cf. [6, 12, 3, 21, 15, 18]):

$$
\begin{gathered}
\frac{\partial \phi}{\partial t}+M \Delta \mu+\nabla \cdot(\phi \mathbf{u})=0 \\
\mu=-\lambda(f(\phi)-\Delta \phi)
\end{gathered}
$$


where $\mu$ is the chemical potential, the parameter $M$ is a mobility constant related to the relaxation time scale, and $f(\phi)=F^{\prime}(\phi)$. The term $M \Delta \mu$ represents the coarse grained form of the microscopic dissipation (general diffusion) relation.

The momentum equation (macroscopic force balance) for the whole system is

$$
\rho\left(\mathbf{u}_{t}+(\mathbf{u} \cdot \nabla) \mathbf{u}\right)=\nabla \cdot \tau
$$

where the total stress $\tau=\nu D(\mathbf{u})-p I+\tau_{e}$ with $D(\mathbf{u})=\nabla \mathbf{u}+\nabla \mathbf{u}^{T}$ and $\tau_{e}$ the extra elastic stress induced by the microscopic internal energy. An energy variational approach is used to derive that

$$
\rho\left(\mathbf{u}_{t}+(\mathbf{u} \cdot \nabla) \mathbf{u}\right)=\nabla \cdot(\nu D(\mathbf{u})-p I-\lambda \nabla \phi \otimes \nabla \phi),
$$

where $p$ includes both the hydrostatic pressure due to the incompressibility and the contributions from the induced stress.

We next use the following identity

$$
\begin{aligned}
\nabla \cdot(\nabla \phi \otimes \nabla \phi) & =(\Delta \phi-f(\phi)) \nabla \phi+\nabla\left(F(\phi)+\frac{1}{2}|\nabla \phi|^{2}\right), \\
& =(\Delta \phi-f(\phi)) \nabla \phi+\nabla\left(F(\phi)+\frac{1}{2}|\nabla \phi|^{2}\right)+\frac{1}{\lambda} \nabla(\phi \mu)-\frac{1}{\lambda} \nabla(\phi \mu)
\end{aligned}
$$

and denote the modified pressure as $\tilde{p}=p+\lambda\left(F(\phi)+\frac{1}{2}|\nabla \phi|^{2}\right)+\frac{1}{\lambda} \phi \mu$ (still denoting it by $p$ for simplicity), then the momentum equation (2.6) can be rewritten as follows

$$
\mathbf{u}_{t}+(\mathbf{u} \cdot \nabla) \mathbf{u}-\nu \nabla \cdot D(\mathbf{u})+\nabla p-\phi \nabla \mu=0
$$

with $\rho=1$ for simplicity.

Combining with the Cahn-Hilliard phase field equation (2.3)-(2.4), the incompressibility constraint $\nabla \cdot \mathbf{u}=0$, and the boundary conditions

$$
\mathbf{n} \cdot \nabla \phi=\mathbf{n} \cdot \nabla \mu=0, \mathbf{u}=\mathbf{0} \text { on } \partial \Omega .
$$

A completed system below is found for the unknown $(\phi, \mu, \mathbf{u}, p)$.

$$
\begin{aligned}
& \frac{\partial \phi}{\partial t}+M \Delta \mu+\nabla \cdot(\phi \mathbf{u})=0, \\
& \mu+\lambda(f(\phi)-\Delta \phi)=0, \\
& \mathbf{u}_{t}+(\mathbf{u} \cdot \nabla) \mathbf{u}-\nu \nabla \cdot D(\mathbf{u})+\nabla p-\phi \nabla \mu=0, \\
& \nabla \cdot \mathbf{u}=0 .
\end{aligned}
$$

We shall adopt the following time discretization developed in [28].

Assuming that $\nu_{1}=\nu_{2}=\nu$. Given initial conditions $\phi^{0}, \mu^{0}, \mathbf{u}^{0}$ and $p^{0}$, we compute $\left(\phi^{k+1}, \mu^{k+1}, \tilde{\mathbf{u}}^{k+1}, \mathbf{u}^{k+1}, p^{k+1}\right)$ for $k \geq 0$ by

$$
\left\{\begin{array}{l}
\frac{\phi^{k+1}-\phi^{k}}{s}+M \Delta \mu^{k+1}+\nabla \cdot\left(\mathbf{u}_{*}^{k} \phi^{k}\right)=0 \\
\mu^{k+1}+\lambda\left(f\left(\phi^{k}\right)-\Delta \phi^{k+1}\right)+\frac{\lambda}{\eta^{2}}\left(\phi^{k+1}-\phi^{k}\right)=0 \\
\left.\mathbf{n} \cdot \nabla \phi^{k+1}\right|_{\partial \Omega}=\left.\mathbf{n} \cdot \nabla \mu^{k+1}\right|_{\partial \Omega}=0
\end{array}\right.
$$

with

$$
\mathbf{u}_{*}^{k}=\mathbf{u}^{k}+s \phi^{k} \nabla \mu^{k+1}
$$




$$
\begin{aligned}
& \left\{\begin{array}{l}
\frac{\tilde{\mathbf{u}}^{k+1}-\mathbf{u}^{k}}{s}-\nu \Delta \tilde{\mathbf{u}}^{k+1}+\nabla p^{k}+\left(\mathbf{u}^{k} \cdot \nabla\right) \tilde{\mathbf{u}}^{k+1}-\phi^{k} \nabla \mu^{k+1}=0, \\
\left.\tilde{\mathbf{u}}^{k+1}\right|_{\partial \Omega}=0
\end{array}\right. \\
& \left\{\begin{array}{l}
\frac{\mathbf{u}^{k+1}-\tilde{\mathbf{u}}^{k+1}}{s}+\nabla\left(p^{k+1}-p^{k}\right)=0, \\
\nabla \cdot \mathbf{u}^{k+1}=0, \\
\left.\mathbf{n} \cdot \mathbf{u}^{k+1}\right|_{\partial \Omega}=0 .
\end{array}\right.
\end{aligned}
$$

The last step can be rewritten as

$$
\left\{\begin{array}{l}
-\Delta\left(p^{k+1}-p^{k}\right)=-\frac{1}{s} \nabla \cdot \tilde{\mathbf{u}}^{k+1} ; \quad \mathbf{u}^{k+1}=\tilde{\mathbf{u}}^{k+1}-s \nabla\left(p^{k+1}-p^{k}\right) . \\
\left.\mathbf{n} \cdot \nabla\left(p^{k+1}-p^{k}\right)\right|_{\partial \Omega}=0
\end{array}\right.
$$

Note that the above scheme is decoupled, linear, and it is shown in [28] that it is unconditionally energy stable.

2.2. Case of non-matched density. Next, we consider the Cahn-Hilliard phase-field model in [2] for mixture of two incompressible fluids with different densities:

$$
\begin{aligned}
& \phi_{t}+M \Delta \mu+\nabla \cdot(\phi \mathbf{u})=0 \\
& \mu+\lambda(f(\phi)-\Delta \phi)=0 \\
& \rho\left(\mathbf{u}_{t}+(\mathbf{u} \cdot \nabla) \mathbf{u}\right)-\mathbf{J} \cdot \nabla \mathbf{u}-\nabla \cdot \nu D(\mathbf{u})+\nabla p-\phi \nabla \mu=0 \\
& \nabla \cdot \mathbf{u}=0
\end{aligned}
$$

with

$$
\mathbf{J}=-\frac{\rho_{1}-\rho_{2}}{2} M \nabla \mu, \rho=\frac{\rho_{1}-\rho_{2}}{2} \phi+\frac{\rho_{1}+\rho_{2}}{2}, \nu=\frac{\nu_{1}-\nu_{2}}{2} \phi+\frac{\nu_{1}+\nu_{2}}{2},
$$

where $\mathbf{u}, p, \rho$ and $\nu$ are the velocity, pressure, density and viscosity of the mixture, respectively.

Equation (2.10) is accompanied with the boundary conditions

$$
\mathbf{n} \cdot \nabla \phi=\mathbf{n} \cdot \nabla \mu=0, \mathbf{u}=\mathbf{0} \text { on } \partial \Omega .
$$

For this case, a totally decoupled, linear, and unconditionally energy stable scheme was constructed in [28]. Below, we present a slightly modified version to fit our need.

$$
\left\{\begin{array}{l}
\frac{\phi^{k+1}-\phi^{k}}{s}+M \Delta \mu^{k+1}+\nabla \cdot\left(\mathbf{u}_{*}^{k} \phi^{k}\right)=0 \\
\mu^{k+1}+\lambda\left(f\left(\phi^{k}\right)-\Delta \phi^{k+1}\right)+\frac{\lambda}{\eta^{2}}\left(\phi^{k+1}-\phi^{k}\right)=0 \\
\left.\mathbf{n} \cdot \nabla \phi^{k+1}\right|_{\partial \Omega}=\left.\mathbf{n} \cdot \nabla \mu^{k+1}\right|_{\partial \Omega}=0
\end{array}\right.
$$

with

$$
\mathbf{u}_{*}^{k}=\mathbf{u}^{k}+s \frac{\phi^{k} \nabla \mu^{k+1}}{\rho^{k}}
$$

$$
\left\{\begin{array}{l}
\rho^{k} \frac{\mathbf{u}^{k+1}-\mathbf{u}^{k}}{s}-\nabla \cdot \nu^{k} \nabla \mathbf{u}^{k+1}+\nabla p^{k}+\rho^{k}\left(\mathbf{u}^{k} \cdot \nabla\right) \mathbf{u}^{k+1}-\mathbf{J}^{k} \cdot \nabla \mathbf{u}^{k+1} \\
-\phi^{k} \nabla \mu^{k+1}=0 \\
\left.\mathbf{u}^{k+1}\right|_{\partial \Omega}=0
\end{array}\right.
$$




$$
\left\{\begin{array}{l}
-\Delta\left(p^{k+1}-p^{k}\right)=-\frac{\chi}{s} \nabla \cdot \mathbf{u}^{k+1} \\
\left.\mathbf{n} \cdot \nabla\left(p^{k+1}-p^{k}\right)\right|_{\partial \Omega}=0
\end{array}\right.
$$

with $\chi=\frac{1}{2} \min \left(\rho_{1}, \rho_{2}\right)$ and

$$
\mathbf{J}^{k}=-\frac{\rho_{1}-\rho_{2}}{2} M \nabla \mu^{k}, \rho^{k}=\frac{\rho_{1}-\rho_{2}}{2} \hat{\phi}^{k}+\frac{\rho_{1}+\rho_{2}}{2}, \nu^{k}=\frac{\nu_{1}-\nu_{2}}{2} \hat{\phi}^{k}+\frac{\nu_{1}+\nu_{2}}{2},
$$

where $\hat{\phi}$ is a cutoff function defined by

$$
\hat{\phi}= \begin{cases}\phi & |\phi| \leq 1 \\ \operatorname{sign}(\phi) & |\phi|>1\end{cases}
$$

\section{Fully Discrete SCHEMES AND ADAPTIVE STRATEGIES}

3.1. Spatial discretization. We first recall notations and summation-by-parts formulae for a second-order finite-difference discretization used in [31,33]. For the sake of brevity, we only present the scheme in two dimensions; the three dimensional case is similar.

Assuming that the computational domain $\Omega=(0, m h) \times(0, n h)$, where $m$ and $n$ are positive integers, and $h>0$ is the grid spacing. The spatial discretization is based on the MAC scheme $[14,22,9]$ for the velocity and pressure. We define

$$
x_{i}=\left(i-\frac{1}{2}\right) h \text { and } y_{j}=\left(j-\frac{1}{2}\right) h,
$$

where $i, j$ can take on integer and half integer values. Consider the following three sets of uniform grid points: (i) east-west edge points $E^{e w}$, (ii) north-south edge points $E^{n s}$, (iii) cell-centered points $C$, and (iv) vertex-centered points $V$, defined via

$$
\begin{aligned}
& E^{e w}=\left\{\left(x_{i+\frac{1}{2}}, y_{j}\right) \mid i=0, \cdots, m ; j=1, \cdots, n\right\}, \\
& E^{n s}=\left\{\left(x_{i}, y_{j+\frac{1}{2}}\right) \mid i=1, \cdots, m ; j=0, \cdots, n\right\}, \\
& \bar{E}^{e w}=\left\{\left(x_{i+\frac{1}{2}}, y_{j}\right) \mid i=0, \cdots, m ; j=0, \cdots, n+1\right\}, \\
& \bar{E}^{n s}=\left\{\left(x_{i}, y_{j+\frac{1}{2}}\right) \mid i=0, \cdots, m+1 ; j=0, \cdots, n\right\}, \\
& C_{m \times n}=\left\{\left(x_{i}, y_{j}\right) \mid i=1, \cdots, m ; j=1, \cdots, n\right\}, \\
& C_{\bar{m} \times n}=\left\{\left(x_{i}, y_{j}\right) \mid i=0, \cdots, m+1 ; j=1, \cdots, n\right\}, \\
& C_{m \times \bar{n}}=\left\{\left(x_{i}, y_{j}\right) \mid i=1, \cdots, m ; j=0, \cdots, n+1\right\}, \\
& C_{\bar{m} \times \bar{n}}=\left\{\left(x_{i}, y_{j}\right) \mid i=0, \cdots, m+1 ; j=0, \cdots, n+1\right\}, \\
& V_{m \times n}=\left\{\left(x_{i+\frac{1}{2}}, y_{j+\frac{1}{2}}\right) \mid i=1, \cdots, m ; j=1, \cdots, n\right\} .
\end{aligned}
$$

We define the function spaces

$$
\begin{array}{lll}
\mathcal{C}_{m \times n}=\left\{\phi: C_{m \times n} \rightarrow \mathbb{R}\right\}, & \mathcal{C}_{\bar{m} \times n}=\left\{\phi: C_{\bar{m} \times n} \rightarrow \mathbb{R}\right\}, \\
\mathcal{C}_{m \times \bar{n}}=\left\{\phi: C_{m \times \bar{n}} \rightarrow \mathbb{R}\right\}, & & \mathcal{C}_{\bar{m} \times \bar{n}}=\left\{\phi: C_{\bar{m} \times \bar{n}} \rightarrow \mathbb{R}\right\}, \\
\mathcal{E}_{m \times n}^{e w}=\left\{u: E^{e w} \rightarrow \mathbb{R}\right\}, & & \mathcal{E}_{m \times n}^{n s}=\left\{v: E^{n s} \rightarrow \mathbb{R}\right\}, \\
\mathcal{E}_{m \times \bar{n}}^{e w}=\left\{u: \bar{E}^{e w} \rightarrow \mathbb{R}\right\}, & & \mathcal{E}_{\bar{m} \times n}^{n s}=\left\{v: \bar{E}^{n s} \rightarrow \mathbb{R}\right\}, \\
\mathcal{V}_{m \times n}=\left\{f: V_{m \times n} \rightarrow \mathbb{R}\right\} . &
\end{array}
$$


Real-valued grid functions with domains $E^{e w}$ are called east-west edge-centered functions and are identified via $f_{i+\frac{1}{2}, j}=f\left(x_{i+\frac{1}{2}}, y_{j}\right)$; those with domains $E^{n s}$ are called north-south edge-centered functions and are identified via $f_{i, j+\frac{1}{2}}=f\left(x_{i}, y_{j+\frac{1}{2}}\right)$; those with domains $C_{m \times n}$ are called cellcentered functions and are identified via $\phi_{i, j}=\phi\left(x_{i}, y_{j}\right)$; and those with domains $V_{m \times n}$ are called vertex-centered functions and are identified via $f_{i+\frac{1}{2}, j+\frac{1}{2}}=f\left(x_{i+\frac{1}{2}}, y_{j+\frac{1}{2}}\right)$. The velocity $\mathbf{u}$ is approximated as edge-centered functions. For example, writing $\mathbf{u}=(u, v), u$ is approximated as an east-west edge-centered function, and $v$ is approximated as a north-south edge-centered function. All other dependent variables are approximated as cell-centered functions.

To complete the spatial discretization, we replace spatial derivatives by finite difference operators. The reader is referred to $[31,33,9]$ for the definitions of the edge-to-center difference operators $d_{x}$ : $\mathcal{E}_{m \times n}^{e w} \rightarrow \mathcal{C}_{m \times n}$ and $d_{y}: \mathcal{E}_{m \times n}^{n s} \rightarrow \mathcal{C}_{m \times n}$; the center-to-edge average and difference operators $A_{x}, D_{x}$ : $\mathcal{C}_{\bar{m} \times n} \rightarrow \mathcal{E}_{m \times n}^{e w}, A_{y}, D_{y}: \mathcal{C}_{m \times \bar{n}} \rightarrow \mathcal{E}_{m \times n}^{n s}$, respectively. The Laplacian operator is approximated to second order by

$$
\nabla_{h}^{2} \phi_{i, j}=\frac{\phi_{i+1, j}+\phi_{i-1, j}+\phi_{i, j+1}+\phi_{i, j-1}-4 \phi_{i, j}}{h^{2}},
$$

where $\phi$ is cell-centered. The Laplacian with non-constant diffusivity/mobility is approximated by

$$
\begin{aligned}
\nabla_{h} \cdot\left(m \nabla_{h} \phi\right)_{i, j} & =\frac{A_{x} m_{i+\frac{1}{2}, j}\left(\phi_{i+1, j}-\phi_{i, j}\right)-A_{x} m_{i-\frac{1}{2}, j}\left(\phi_{i, j}-\phi_{i-1, j}\right)}{h^{2}} \\
& +\frac{A_{y} m_{i, j+\frac{1}{2}}\left(\phi_{i, j+1}-\phi_{i, j}\right)-A_{y} m_{i, j-\frac{1}{2}}\left(\phi_{i, j}-\phi_{i, j-1}\right)}{h^{2}},
\end{aligned}
$$

where both $\phi$ and $m$ are assumed to be cell-centered, and $A_{x}$ and $A_{y}$ are the averaging operators defined component-wise as

$$
\begin{aligned}
& A_{x} m_{i+\frac{1}{2}, j}=\frac{m_{i+1, j}+m_{i, j}}{2}, A_{x} m_{i-\frac{1}{2}, j}=\frac{m_{i, j}+m_{i-1, j}}{2} \\
& A_{y} m_{i, j+\frac{1}{2}}=\frac{m_{i, j+1}+m_{i, j}}{2}, A_{y} m_{i, j-\frac{1}{2}}=\frac{m_{i, j}+m_{i, j-1}}{2} .
\end{aligned}
$$

The grid function norms are defined as follows [31, 33, 9]: for $\phi \in \mathcal{C}_{m \times n}, 1 \leq p<\infty$,

$$
\|\phi\|_{p}=\left(h^{2} \sum_{i=1}^{m} \sum_{j=1}^{n}\left|\phi_{i, j}\right|^{p}\right)^{1 / p} .
$$

For any $\phi \in \mathcal{C}_{\bar{m} \times \bar{n}}$, we define

$$
\|\nabla \phi\|_{2}=\sqrt{h^{2}\left[D_{x} \phi \| D_{x} \phi\right]_{e w}+h^{2}\left[D_{y} \phi \| D_{y} \phi\right]_{n s}} .
$$

For the edge-centered cell velocity $\mathbf{u}$, we define

$$
\|\mathbf{u}\|_{2}=\sqrt{h^{2}[u \| u]_{e w}+h^{2}[v \| v]_{n s}},
$$

where $u \in \mathcal{E}_{m \times n}^{e w}$, and $v \in \mathcal{E}_{m \times n}^{n s}$.

We recall (cf. [31, 33, 9]) the following summation-by-parts formulae which are essential to prove the stability of the fully discrete schemes.

Proposition 3.1. (Summation-by-parts): If $\phi \in \mathcal{C}_{\bar{m} \times n} \cup \mathcal{C}_{\bar{m} \times \bar{n}}$ and $f \in \mathcal{E}_{m \times n}^{e w}$, then

$$
h^{2}\left[D_{x} \phi \| f\right]_{e w}=-h^{2}\left(\phi \| d_{x} f\right)-h\left(A_{x} \phi_{\frac{1}{2}, *} \mid f_{\frac{1}{2}, *}\right)+h\left(A_{x} \phi_{m+\frac{1}{2}, *} \mid f_{m+\frac{1}{2}, *}\right),
$$

and if $\phi \in \mathcal{C}_{m \times \bar{n}} \cup \mathcal{C}_{\bar{m} \times \bar{n}}$ and $f \in \mathcal{E}_{m \times n}^{n s}$, then

$$
h^{2}\left[D_{y} \phi \| f\right]_{n s}=-h^{2}\left(\phi \| d_{y} f\right)-h\left(A_{y} \phi_{*, \frac{1}{2}} \mid f_{*, \frac{1}{2}}\right)+h\left(A_{y} \phi_{*, n+\frac{1}{2}} \mid f_{*, n+\frac{1}{2}}\right) .
$$


If $f \in \mathcal{V}_{m \times n}$ and $g \in \mathcal{E}_{\bar{m} \times n}^{n s}$, then

$$
h^{2}\left[d_{x} f \| g\right]_{n s}=-h^{2}<f \| D_{x} g>-h\left[A_{x} g_{\frac{1}{2}, *} \mid f_{\frac{1}{2}, *}\right]+h\left[A_{x} g_{m+\frac{1}{2}, *} \mid f_{m+\frac{1}{2}, *}\right],
$$

and if $f \in \mathcal{V}_{m \times n}$ and $g \in \mathcal{E}_{m \times \bar{n}}^{e w}$, then

$$
h^{2}\left[d_{y} f \| g\right]_{e w}=-h^{2}<f \| D_{y} g>-h\left[A_{y} g_{*, \frac{1}{2}} \mid f_{*, \frac{1}{2}}\right]+h\left[A_{y} g_{*, m+\frac{1}{2}} \mid f_{*, n+\frac{1}{2}}\right] .
$$

3.2. Fully discrete scheme and its stability analysis. Now we are ready to present the fullydiscrete version of the scheme (2.8a) - (2.8c) and (2.9).

Given $\phi^{k}, \mu^{k}, p^{k} \in \mathcal{C}_{\bar{m} \times \bar{n}}, u^{k} \in \mathcal{E}_{m \times n}^{e w}$, and $v^{k} \in \mathcal{E}_{m \times n}^{n s}$, find $\phi^{k+1}, \mu^{k+1}, p^{k+1} \in \mathcal{C}_{\bar{m} \times \bar{n}}, \tilde{u}^{k+1} \in \mathcal{E}_{m \times n}^{e w}$, $\tilde{v}^{k+1} \in \mathcal{E}_{m \times n}^{n s}, u^{k+1} \in \mathcal{E}_{m \times n}^{e w}$, and $v^{k+1} \in \mathcal{E}_{m \times n}^{n s}$ such that

$$
\begin{aligned}
\phi^{k+1}-\phi^{k} & =-s M\left(d_{x}\left(D_{x} \mu^{k+1}\right)+d_{y}\left(D_{y} \mu^{k+1}\right)\right)-s\left(d_{x}\left(A_{x} \phi^{k} u_{*}^{k}\right)+d_{y}\left(A_{y} \phi^{k} v_{*}^{k}\right)\right), \\
\mu^{k+1} & =-\lambda\left(f\left(\phi^{k}\right)-\Delta_{h} \phi^{k+1}\right)-\frac{\lambda}{\eta^{2}}\left(\phi^{k+1}-\phi^{k}\right), \\
\tilde{u}^{k+1}-u_{*}^{k} & =s \nu\left(d_{y}\left(D_{y} \tilde{u}^{k+1}\right)+D_{x}\left(d_{x} \tilde{u}^{k+1}\right)\right)-\frac{s}{2}\left(d_{x} u^{k}+d_{y} v^{k}\right) \tilde{u}^{k+1} \\
& -s D_{x} p^{k}-s\left(u^{k} D_{x} \tilde{u}^{k+1}+v^{k} D_{y} \tilde{u}^{k+1}\right), \\
\tilde{v}^{k+1}-v_{*}^{k} & =s \nu\left(d_{x}\left(D_{x} \tilde{v}^{k+1}\right)+D_{y}\left(d_{y} \tilde{v}^{k+1}\right)\right)-\frac{s}{2}\left(d_{x} u^{k}+d_{y} v^{k}\right) \tilde{v}^{k+1} \\
& -s D_{y} p^{k}-s\left(u^{k} D_{x} \tilde{v}^{k+1}+v^{k} D_{y} \tilde{v}^{k+1}\right), \\
-\Delta_{h}\left(p^{k+1}-p^{k}\right) & =-\frac{1}{s} \nabla_{h} \cdot \tilde{\mathbf{u}}^{k+1}, \\
u^{k+1}-\tilde{u}^{k+1} & =-s D_{x}\left(p^{k+1}-p^{k}\right), \\
v^{k+1}-\tilde{v}^{k+1} & =-s D_{y}\left(p^{k+1}-p^{k}\right),
\end{aligned}
$$

where

$$
\begin{aligned}
u_{*}^{k} & =u^{k}+s \phi^{k} D_{x} \mu^{k+1}, \\
v_{*}^{k} & =v^{k}+s \phi^{k} D_{y} \mu^{k+1},
\end{aligned}
$$

and the boundary conditions are

$$
\mathbf{n} \cdot \nabla \phi^{k+1}=\mathbf{n} \cdot \nabla \mu^{k+1}=0, \tilde{\mathbf{u}}^{k+1}=\mathbf{0}, \quad \mathbf{n} \cdot \mathbf{u}^{k+1}=0 \text { on } \partial \Omega .
$$

We note that the pressure Poisson equation (3.7e) is discretized using the cell centered approximation (3.1), and the Poisson type equations (3.7c) and (3.7d) for the velocity components are discretized using the edge/face-centered approximation which is described below:

$$
\begin{aligned}
\tilde{u}_{i+1 / 2, j}^{k+1}-s \nu \Delta \tilde{u}_{i+1 / 2, j}^{k+1} & =u_{i+1 / 2, j}^{k}-s\left(\left(p_{x}\right)_{i, j}^{k}+\left(p_{x}\right)_{i+1, j}^{k}\right) / 2-s\left(u^{k} \tilde{u}_{x}^{k+1}+v^{k} \tilde{u}_{y}^{k+1}\right)_{i+1 / 2, j} \\
& +s\left(\left(\phi^{k}\left(\mu_{x}\right)^{k+1}\right)_{i, j}+\left(\phi^{k}\left(\mu_{x}\right)^{k+1}\right)_{i+1, j}\right) / 2,
\end{aligned}
$$

and

$$
\begin{aligned}
\tilde{v}_{i, j+1 / 2}^{k+1}-s \nu \Delta \tilde{v}_{i, j+1 / 2}^{k+1} & =v_{i, j+1 / 2}^{k}-s\left(\left(p_{y}\right)_{i, j}^{k}+\left(p_{y}\right)_{i, j+1}^{k}\right) / 2-s\left(u^{k} \tilde{v}_{x}^{k+1}+v^{k} \tilde{v}_{y}^{k+1}\right)_{i, j+1 / 2} \\
& +s\left(\left(\phi^{k}\left(\mu_{y}\right)^{k+1}\right)_{i, j}+\left(\phi^{k}\left(\mu_{y}\right)^{k+1}\right)_{i, j+1}\right) / 2 .
\end{aligned}
$$


Theorem 3.1. Assume that the potential function $F(\phi)$ satisfying $\max _{|\phi| \in \mathbb{R}}\left|F^{\prime \prime}(\phi)\right| \leq \frac{2}{\eta^{2}}$, then the scheme (3.7) is unconditionally stable, and satisfies the following discrete energy law:

$$
\begin{aligned}
\frac{1}{2}\left\|u^{k+1}\right\|_{2}^{2}+\frac{\lambda}{2}\left\|\nabla \phi^{k+1}\right\|_{2}^{2} & +\lambda\left(F\left(\phi^{k+1}\right) \mid 1\right)+\frac{s^{2}}{2}\left\|\nabla p^{k+1}\right\|_{2}^{2}+s M\left\|\nabla \mu^{k+1}\right\|_{2}^{2}+s \nu\left\|\nabla \tilde{\mathbf{u}}^{k+1}\right\|_{2}^{2} \\
& \leq \frac{1}{2}\left\|u^{k}\right\|_{2}^{2}+\frac{\lambda}{2}\left\|\nabla \phi^{k}\right\|_{2}^{2}+\lambda\left(F\left(\phi^{k}\right) \mid 1\right)+\frac{s^{2}}{2}\left\|\nabla p^{k}\right\|_{2}^{2}
\end{aligned}
$$

Proof. Take the discrete inner-product of (3.7c) with $2 \tilde{u}^{k+1}$ and of (3.7d) with $2 \tilde{v}^{k+1}$, respectively. Using Proposition 3.1 and the following property

$$
\begin{aligned}
& {\left[u^{k} D_{x} \tilde{u}^{k+1}+v^{k} D_{y} \tilde{u}^{k+1} \| \tilde{u}^{k+1}\right]_{e w}+\left[u^{k} D_{x} \tilde{v}^{k+1}+v^{k} D_{y} \tilde{v}^{k+1} \| \tilde{v}^{k+1}\right]_{n s}} \\
& +\frac{1}{2}\left[\left(d_{x} u^{k}+d_{y} v^{k}\right) \tilde{u}^{k+1} \| \tilde{u}^{k+1}\right]_{e w}+\frac{1}{2}\left[\left(d_{x} u^{k}+d_{y} v^{k}\right) \tilde{v}^{k+1} \| \tilde{v}^{k+1}\right]_{n s}=0,
\end{aligned}
$$

we derive

$$
\begin{aligned}
& {\left[\tilde{u}^{k+1} \| \tilde{u}^{k+1}\right]_{e w}+\left[\tilde{v}^{k+1} \| \tilde{v}^{k+1}\right]_{n s}-\left[u_{*}^{k} \| u_{*}^{k}\right]_{e w}-\left[v_{*}^{k} \| v_{*}^{k}\right]_{n s}+\left[\tilde{u}^{k+1}-u_{*}^{k} \| \tilde{u}^{k+1}-u_{*}^{k}\right]_{e w}} \\
& +\left[\tilde{v}^{k+1}-v_{*}^{k} \| \tilde{v}^{k+1}-v_{*}^{k}\right]_{n s}=-2 s \nu\left(<D_{y} \tilde{u}^{k+1}\left\|D_{y} \tilde{u}^{k+1}>+<D_{x} \tilde{v}^{k+1}\right\| D_{x} \tilde{v}^{k+1}>\right) \\
& -2 s \nu\left(\left(d_{x} \tilde{u}^{k+1} \| d_{x} \tilde{u}^{k+1}\right)+\left(d_{y} \tilde{v}^{k+1} \| d_{y} \tilde{v}^{k+1}\right)\right)-2 s\left(\left[D_{x} p^{k+1} \| \tilde{u}^{k+1}\right]_{e w}+\left[D_{y} p^{k+1} \| \tilde{v}^{k+1}\right]_{n s}\right) .
\end{aligned}
$$

Taking the discrete inner-product of (3.7f) with $2 D_{x} p^{k}$ and of $(3.7 \mathrm{~g})$ with $2 D_{y} p^{k}$, respectively, we find

$$
\begin{aligned}
& s\left(\left[D_{x} p^{k+1} \| D_{x} p^{k+1}\right]_{e w}+\left[D_{y} p^{k+1} \| D_{y} p^{k+1}\right]_{n s}-\left[D_{x} p^{k} \| D_{x} p^{k}\right]_{e w}-\left[D_{y} p^{k} \| D_{y} p^{k}\right]_{n s}\right. \\
& \left.-\left[D_{x} p^{k+1}-D_{x} p^{k} \| D_{x} p^{k+1}-D_{x} p^{k}\right]_{e w}-\left[D_{y} p^{k+1}-D_{y} p^{k} \| D_{y} p^{k+1}-D_{y} p^{k}\right]_{n s}\right) \\
& =2\left[\tilde{u}^{k+1} \| D_{x} p^{k}\right]_{e w}+2\left[\tilde{v}^{k+1} \| D_{y} p^{k}\right]_{n s .}
\end{aligned}
$$

From Eqs. (3.7f) and (3.7g), we derive that

$$
\begin{aligned}
{\left[u^{k+1}-\tilde{u}^{k+1} \| u^{k+1}-\tilde{u}^{k+1}\right]_{e w} } & =s^{2}\left[D_{x}\left(p^{k+1}-p^{k}\right) \| D_{x}\left(p^{k+1}-p^{k}\right)\right]_{e w}, \\
{\left[v^{k+1}-\tilde{v}^{k+1} \| v^{k+1}-\tilde{v}^{k+1}\right]_{n s} } & =s^{2}\left[D_{y}\left(p^{k+1}-p^{k}\right) \| D_{y}\left(p^{k+1}-p^{k}\right)\right]_{n s} .
\end{aligned}
$$

Taking the discrete inner-product of (3.7f) with $u^{k+1}$ and of $(3.7 \mathrm{~g})$ with $v^{k+1}$, respectively, we obtain

$$
\begin{aligned}
& {\left[u^{k+1} \| u^{k+1}\right]_{e w}+\left[v^{k+1} \| v^{k+1}\right]_{n s}+\left[u^{k+1}-\tilde{u}^{k+1} \| u^{k+1}-\tilde{u}^{k+1}\right]_{e w}} \\
& +\left[v^{k+1}-\tilde{v}^{k+1} \| v^{k+1}-\tilde{v}^{k+1}\right]_{n s}-\left[\tilde{u}^{k+1} \| \tilde{u}^{k+1}\right]_{e w}-\left[\tilde{v}^{k+1} \| \tilde{v}^{k+1}\right]_{n s}=0 .
\end{aligned}
$$

Combining the four equalities above, we find

$$
\begin{aligned}
& -\left[u_{*}^{k} \| u_{*}^{k}\right]_{e w}-\left[v_{*}^{k} \| v_{*}^{k}\right]_{n s}+\left[\tilde{u}^{k+1}-u_{*}^{k} \| \tilde{u}^{k+1}-u_{*}^{k}\right]_{e w}+\left[\tilde{v}^{k+1}-v_{*}^{k} \| \tilde{v}^{k+1}-v_{*}^{k}\right]_{n s} \\
& +\left[u^{k+1} \| u^{k+1}\right]_{e w}+\left[v^{k+1} \| v^{k+1}\right]_{n s}+s^{2}\left(\left[D_{x}\left(p^{k+1}-p^{k}\right) \| D_{x}\left(p^{k+1}-p^{k}\right)\right]_{e w}\right. \\
& \left.+\left[D_{y}\left(p^{k+1}-p^{k}\right) \| D_{y}\left(p^{k+1}-p^{k}\right)\right]_{n s}-\left[D_{x} p^{k} \| D_{x} p^{k}\right]_{e w}-\left[D_{y} p^{k} \| D_{y} p^{k}\right]_{n s}\right) \\
& +2 s \nu\left(<D_{y} \tilde{u}^{k+1}\left\|D_{y} \tilde{u}^{k+1}>+<D_{x} \tilde{v}^{k+1}\right\| D_{x} \tilde{v}^{k+1}>\right)+2 s \nu\left(\left(d_{x} \tilde{u}^{k+1} \| d_{x} \tilde{u}^{k+1}\right)\right. \\
& \left.+\left(d_{y} \tilde{v}^{k+1} \| d_{y} \tilde{v}^{k+1}\right)\right)=0 .
\end{aligned}
$$

Next, we use (3.7h) and (3.7i) to deal with the term $-\left[u_{*}^{k} \| u_{*}^{k}\right]_{e w}-\left[v_{*}^{k} \| v_{*}^{k}\right]_{n s}$ above. 
Taking the discrete inner-product of $(3.7 \mathrm{~h})$ with $2 u_{*}^{k}$ and of (3.7i) with $2 v_{*}^{k}$, respectively, gives us

$$
\begin{aligned}
& {\left[u_{*}^{k} \| u_{*}^{k}\right]_{e w}+\left[v_{*}^{k} \| v_{*}^{k}\right]_{n s}-\left[u^{k} \| u^{k}\right]_{e w}-\left[v^{k} \| v^{k}\right]_{n s}+\left[u_{*}^{k}-u^{k} \| u_{*}^{k}-u^{k}\right]_{e w}+\left[v_{*}^{k}-v^{k} \| v_{*}^{k}-v^{k}\right]_{n s}} \\
& =2 s\left[A_{x} \phi^{k} D_{x} \mu^{k+1} \| u_{*}^{k}\right]+2 s\left[A_{y} \phi^{k} D_{y} \mu^{k+1} \| v_{*}^{k}\right] .
\end{aligned}
$$

Summing up the two equalities above, we obtain

$$
\begin{aligned}
& {\left[u^{k+1} \| u^{k+1}\right]_{e w}+\left[v^{k+1} \| v^{k+1}\right]_{n s}-\left[u^{k} \| u^{k}\right]_{e w}-\left[v^{k} \| v^{k}\right]_{n s}+\left[u_{*}^{k}-u^{k} \| u_{*}^{k}-u^{k}\right]_{e w}} \\
& +\left[v_{*}^{k}-v^{k} \| v_{*}^{k}-v^{k}\right]_{n s}+\left[\tilde{u}^{k+1}-u_{*}^{k} \| \tilde{u}^{k+1}-u_{*}^{k}\right]_{e w} \\
& +\left[\tilde{v}^{k+1}-v_{*}^{k} \| \tilde{v}^{k+1}-v_{*}^{k}\right]_{n s}+s^{2}\left(\left[D_{x}\left(p^{k+1}-p^{k}\right) \| D_{x}\left(p^{k+1}-p^{k}\right)\right]_{e w}\right. \\
& \left.+\left[D_{y}\left(p^{k+1}-p^{k}\right) \| D_{y}\left(p^{k+1}-p^{k}\right)\right]_{n s}-\left[D_{x} p^{k} \| D_{x} p^{k}\right]_{e w}-\left[D_{y} p^{k} \| D_{y} p^{k}\right]_{n s}\right) \\
& +2 s \nu\left(<D_{y} \tilde{u}^{k+1}\left\|D_{y} \tilde{u}^{k+1}>+<D_{x} \tilde{v}^{k+1}\right\| D_{x} \tilde{v}^{k+1}>\right) \\
& +2 s \nu\left(\left(d_{x} \tilde{u}^{k+1} \| d_{x} \tilde{u}^{k+1}\right)+\left(d_{y} \tilde{v}^{k+1} \| d_{y} \tilde{v}^{k+1}\right)\right) \\
& =2 s\left[A_{x} \phi^{k} D_{x} \mu^{k+1} \| u_{*}^{k}\right]+2 s\left[A_{y} \phi^{k} D_{y} \mu^{k+1} \| v_{*}^{k}\right] .
\end{aligned}
$$

Next, we take the discrete inner-product of (3.7a) with $-2 \mu^{k+1}$ to get

$$
\begin{aligned}
& -2\left(\phi^{k+1}-\phi^{k} \| \mu^{k+1}\right)=-2 s\left(\left[D_{x} \mu^{k+1} \| D_{x} \mu^{k+1}\right]_{e w}+\left[D_{y} \mu^{k+1} \| D_{y} \mu^{k+1}\right]_{n s}\right) \\
& +2 s\left(\left[A_{x} \phi^{k} u_{*}^{k} \| D_{x} \mu^{k+1}\right]_{e w}+\left[A_{y} \phi^{k} v_{*}^{k} \| D_{y} \mu^{k+1}\right]_{n s}\right) .
\end{aligned}
$$

Taking the discrete inner-product of (3.7b) with $2\left(\phi^{k+1}-\phi^{k}\right)$, we find

$$
\begin{aligned}
& 2\left(\mu^{k+1} \| \phi^{k+1}-\phi^{k}\right)=-2 \lambda\left(f\left(\phi^{k} \| \phi^{k+1}-\phi^{k}\right)-\lambda\left(\left[D_{x} \phi^{k+1} \| D_{x} \phi^{k+1}\right]_{e w}\right.\right. \\
& +\left[D_{y} \phi^{k+1} \| D_{y} \phi^{k+1}\right]_{n s}-\left[D_{x} \phi^{k} \| D_{x} \phi^{k}\right]_{e w}-\left[D_{y} \phi^{k} \| D_{y} \phi^{k}\right]_{n s} \\
& +\left[D_{x} \phi^{k+1}-D_{x} \phi^{k} \| D_{x} \phi^{k+1}-D_{x} \phi^{k}\right]_{e w}+\left[D_{y} \phi^{k+1}-D_{y} \phi^{k} \| D_{y} \phi^{k+1}-D_{y} \phi^{k}\right]_{n s} \\
& -\frac{2 \lambda}{\eta^{2}}\left(\phi^{k+1}-\phi^{k} \| \phi^{k+1}-\phi^{k}\right) .
\end{aligned}
$$

The Taylor expansion for $F\left(\phi^{k+1}\right)$ shows that

$$
F\left(\phi^{k+1}\right)=F\left(\phi^{k}\right)+f\left(\phi^{k}\right)\left(\phi^{k+1}-\phi^{k}\right)+\frac{f^{\prime}\left(\xi^{k}\right)}{2}\left(\phi^{k+1}-\phi^{k}\right)^{2} .
$$

Combining Eqs. (3.10)-(3.13), using the assumption that $\max _{|\phi| \in \mathbb{R}}\left|F^{\prime \prime}(\phi)\right| \leq \frac{2}{\eta^{2}}$, we have

$$
\begin{aligned}
& \left\|\mathbf{u}^{k+1}\right\|_{2}^{2}-\left\|\mathbf{u}^{k}\right\|_{2}^{2}+\left\|\mathbf{u}_{*}^{k}-\mathbf{u}^{k}\right\|_{2}^{2}+\left\|\tilde{\mathbf{u}}^{k+1}-\mathbf{u}_{*}^{k}\right\|_{2}^{2}+s^{2}\left(\left\|\nabla p^{k+1}\right\|_{2}^{2}-\left\|\nabla p^{k}\right\|_{2}^{2}\right. \\
& +2 s \nu\left\|\nabla \tilde{\mathbf{u}}^{k+1}\right\|_{2}^{2}+2 s M\left\|\nabla \mu^{k+1}\right\|_{2}^{2}+2 \lambda\left(F\left(\phi^{k+1}\right)-F\left(\phi^{k}\right) \mid 1\right) \\
& +\lambda\left(\left\|\nabla \phi^{k+1}\right\|_{2}^{2}-\left\|\nabla \phi^{k}\right\|_{2}^{2}+\left\|\nabla \phi^{k+1}-\nabla \phi^{k}\right\|_{2}^{2}\right) \leq 0
\end{aligned}
$$

which implies the desire result. 
Similarly, we construct the fully discrete version of $(2.12 \mathrm{a})-(2.12 \mathrm{~d})$ as follows:

$$
\begin{aligned}
& \phi^{k+1}-\phi^{k}=-s M\left(d_{x}\left(D_{x} \mu^{k+1}\right)+d_{y}\left(D_{y} \mu^{k+1}\right)\right)-s\left(d_{x}\left(A_{x} \phi^{k} u_{*}^{k}\right)+d_{y}\left(A_{y} \phi^{k} v_{*}^{k}\right)\right) \\
& \mu^{k+1}=-\lambda\left(f\left(\phi^{k}\right)-\Delta_{h} \phi^{k+1}\right)-\frac{\lambda}{\eta^{2}}\left(\phi^{k+1}-\phi^{k}\right) \\
&\left(A_{x} \rho^{k}\right) u^{k+1}-s \nabla \cdot\left(\left(A_{x} \nu^{k}\right) \nabla_{h} u^{k+1}\right)=\left(A_{x} \rho^{k}\right) u^{k}-s D_{x} p^{k}-s\left(A_{x} \rho^{k}\right)\left(u^{k} u_{x}^{k+1}+v^{k} u_{y}^{k+1}\right) \\
&-s \frac{\rho_{1}-\rho_{2}}{2} M \nabla_{h} \mu^{k} \cdot \nabla_{h} u^{k+1}+s\left(A_{x} \phi^{k}\right) D_{x} \mu^{k+1}, \\
&\left(A_{y} \rho^{k}\right) v^{k+1}-s \nabla_{h} \cdot\left(\left(A_{y} \nu^{k}\right) \nabla_{h} v^{k+1}\right)=\left(A_{y} \rho^{k}\right) v^{k}-s D_{y} p^{k}-s\left(A_{y} \rho^{k}\right)\left(u^{k} v_{x}^{k+1}+v^{k} v_{y}^{k+1}\right) \\
& \quad-s \frac{\rho_{1}-\rho_{2}}{2} M \nabla_{h} \mu^{k} \cdot \nabla_{h} v^{k+1}+s\left(A_{y} \phi^{k}\right) D_{y} \mu^{k+1} \\
&-\Delta_{h}\left(p^{k+1}-p^{k}\right)=-\frac{\chi}{s} \nabla_{h} \cdot \mathbf{u}^{k+1}
\end{aligned}
$$

with the boundary conditions

$$
\mathbf{n} \cdot \nabla \phi^{k+1}=\mathbf{n} \cdot \nabla \mu^{k+1}=0, \tilde{\mathbf{u}}^{k+1}=\mathbf{0}, \quad \mathbf{n} \cdot \mathbf{u}^{k+1}=0 \text { on } \partial \Omega .
$$

It can be shown, by combining the stability proof in [28] and the argument in the proof of Theorem 3.1 , that the above scheme is also unconditionally energy stable under the same assumption. We omit the detail for the sake of brevity.

3.3. Space and time adaptive strategy. The fully discrete schemes (3.7) and (3.14) lead to a sequence of discrete elliptic equations at each time step. We shall solve them by using the adaptive multigrid method developed in [34, 35, 32, 8]. In fact, since the system of governing equations is discretized on a block-structured Cartesian mesh, the composite mesh consists of a hierarchy of levels which is particularly suitable for adaptive multigrid. At each time step, we check grid cells for refinement using a simple undivided gradient test. Since it is essential that much more refinement is needed in the diffuse interface region. This test marks grid cells where the finite differences of the phase-field functions are large. In particular, the set of cell-centered points is marked for refinement if

$$
\sqrt{\left(\phi_{i+1, j}-\phi_{i-1, j}\right)^{2}+\left(\phi_{i, j+1}-\phi_{i, j-1}\right)^{2}}>C_{k},
$$

where $C_{k}$ is the critical value for level $k$. More sophisticated mesh refinement strategy could also be used here. For instance, curvature-based mesh refinement criteria [29]. For a detailed discussion of all the aspects of the adaptive algorithm, the reader is referred to [34].

In our case, the refinement takes place around the interface of two mixtures, so as to have sufficient resolution of the interface, see Fig. 4.1 (right).

The schemes (3.7) and (3.14) are shown to be unconditionally energy stable, which allows us to use large time step sizes whenever suitable. In order to maintain the desired accuracy, we adjust the time steps based on the total energy derivative as follows (cf. [23, 37]):

$$
\Delta t^{n+1}=\max \left(\Delta t_{\min }, \frac{\Delta t_{\max }}{\sqrt{1+\alpha\left|\frac{E\left(t^{n}\right)-E\left(t^{n-1)}\right.}{\Delta t^{n}}\right|}}\right),
$$

where $\Delta t_{\max }$ and $\Delta t_{\min }$ are a preset upper and lower bound for the time step sizes, and $\alpha$ is an adjustable constant. We observe from (3.15) that large (resp. small) deviation in total energy will yield small (resp. large) time step size. 
3.4. Second-order scheme. The schemes (2.8) and (2.12), and their fully discrete versions (3.7) and (3.14), are only first-order accurate in time. It is highly desirable to have a second-order-intime scheme, which inherits all essential advantages of the first-order schemes, to match accuracy of the second-order-in-space finite-difference method. Very recently, Han and Wang [13] developed a second order in time numerical scheme for Cahn-Hilliard-Navier-Stokes phase field model with matched density. The scheme is based on second order convex-splitting for the Cahn-Hilliard equation and pressure-projection for the Navier-Stokes equation. The pressure is decoupled from the velocity and phase field, but the velocity field is still coupled with the phase field. Here we construct below a second-order scheme based on the improved Euler method (also known as Heun method). In our schemes the velocity filed is decoupled from the phase field.

For the sake of brevity, we will only consider the case with matched density.

Step 1. Given the solution $\left(\phi^{k}, \mu^{k}, \tilde{\mathbf{u}}^{k}, \mathbf{u}^{k}\right.$ and $\left.p^{k}\right)$ at step $n$, We first compute $\phi^{k+1}, \mu^{k+1}, \tilde{\mathbf{u}}^{k+1}$, $\mathbf{u}^{k+1}$ and $p^{k+1}$ from $(2.8 \mathrm{a})-(2.8 \mathrm{c})$ and $(2.9)$, and rename them as $\hat{\phi}^{k+1}, \hat{\mu}^{k+1}, \tilde{\mathbf{u}}^{k+1}, \hat{\mathbf{u}}^{k+1}$ and $\hat{p}^{k+1}$, respectively.

Step 2. Compute $\left(\phi^{k+1}, \mu^{k+1}, \tilde{\tilde{\mathbf{u}}}^{k+1}, \mathbf{u}^{k+1}, p^{k+1}\right)$ as follows:

$$
\left\{\begin{array}{l}
\frac{\phi^{k+1}-\phi^{k}}{s}+M \Delta \frac{\mu^{k+1}+\mu^{k}}{2}+\nabla \cdot\left(\frac{\hat{\mathbf{u}}_{*}^{k+1} \hat{\phi}^{k+1}+\mathbf{u}_{*}^{k} \phi^{k}}{2}\right)=0 \\
\frac{\mu^{k+1}+\mu^{k}}{2}+\lambda\left(f\left(\frac{\hat{\phi}^{k+1}+\phi^{k}}{2}\right)-\Delta \frac{\phi^{k+1}+\phi^{k}}{2}\right)+\frac{s \lambda}{\eta^{2}}\left(\phi^{k+1}-\phi^{k}\right)=0 \\
\left.\mathbf{n} \cdot \nabla \phi^{k+1}\right|_{\partial \Omega}=\left.\mathbf{n} \cdot \nabla \mu^{k+1}\right|_{\partial \Omega}=0
\end{array}\right.
$$

with

$$
\begin{aligned}
& \hat{\mathbf{u}}_{*}^{k+1}=\hat{\mathbf{u}}^{k+1}+s \hat{\phi}^{k+1} \nabla \hat{\mu}^{k+1}, \\
& \mathbf{u}_{*}^{k}=\mathbf{u}^{k}+s \phi^{k} \nabla \mu^{k+1} \text {; } \\
& \left\{\begin{array}{l}
\frac{\tilde{\tilde{\mathbf{u}}}^{k+1}-\mathbf{u}^{k}}{s}-\nu \Delta \frac{\tilde{\tilde{\mathbf{u}}}^{k+1}+\tilde{\tilde{\mathbf{u}}}^{k}}{2}+\nabla p^{k}+\left(\frac{\hat{\mathbf{u}}^{k+1}+\mathbf{u}^{k}}{2} \cdot \nabla\right) \frac{\tilde{\tilde{\mathbf{u}}}^{k+1}+\tilde{\tilde{\mathbf{u}}}^{k}}{2} \\
-\frac{\hat{\phi}^{k+1}+\phi^{k}}{2} \nabla \frac{\mu^{k+1}+\mu^{k}}{2}=0, \\
\left.\tilde{\tilde{\mathbf{u}}}^{k+1}\right|_{\partial \Omega}=0 ;
\end{array}\right. \\
& \left\{\begin{array}{l}
\frac{\mathbf{u}^{k+1}-\tilde{\tilde{\mathbf{u}}}^{k+1}}{s}+\frac{1}{2} \nabla\left(p^{k+1}-p^{k}\right)=0, \\
\nabla \cdot \mathbf{u}^{k+1}=0, \\
\left.\mathbf{n} \cdot \mathbf{u}^{k+1}\right|_{\partial \Omega}=0 .
\end{array}\right.
\end{aligned}
$$

The fully discrete version of the above scheme can be constructed similarly as (3.7).

\section{NumERICAL RESUlts}

We present in this section some numerical experiments in two dimensions using the schemes constructed above.

For the examples with matched density, the computational domain is $[-1,1] \times[-1,1]$. For adaptive refinement, we choose the root-level grid size to be $32 \times 32$, and perform three levels of 
refinement, i.e., the finest mesh size is $h=2 / 256$. We set time step size $\Delta t=0.01$, which is sufficient for the purpose of accuracy, see Fig. 4.1 (left). The other parameters are

$$
M=0.002, \lambda=0.01, \nu=1, \eta=0.02 .
$$

For the examples with variable density, the computational domain is $[0,1] \times[0,1.5]$. The rootlevel grid size is $128 \times 192$, and three levels of refinement are used, i.e., the finest mesh size is $h=1 / 1024$. The parameters will be given in section 4.3 later.

4.1. Example 1. Surface tension effect. Since it is not an easy matter to rigorously prove that the scheme (3.16a)-(3.16e) is energy stable and second-order accurate, we shall provide some numerical evidence to show that it is indeed the case.

We take $\rho_{1}=\rho_{2}=1, \nu_{1}=\nu_{2}=1$. We set the initial velocity and pressure to be zero, and start with a square shaped fluid bubble in the domain $[-1,1] \times[-1,1]$. It is clear that the square will eventually evolve into a circle due to the surface tension. In Fig. 4.3, characteristic snapshots are shown at different times.

In the left of Fig. 4.2, we plot the total energy which is shown to be decreasing as the system evolves. This indicates that the second-order scheme is indeed energy stable.

In the next we use the same example above to test the convergence rate of the scheme (3.16a)(3.16e). The mesh spacings are taken to be $h=\frac{2}{64}, \frac{2}{128}, \frac{2}{256}, \frac{2}{512}, \frac{2}{1024}$, each one half the size of the prevours. We take the linear refinement path $s=0.0256 h$. The error between two different grid spacings $\phi^{h}$ and $\phi^{h / 2}$ is calculated by

$$
e_{i, j}^{h: h / 2}=\phi_{i, j}^{h}-\frac{1}{4}\left(\phi_{2 i, 2 j}^{h / 2}+\phi_{2 i-1,2 j}^{h / 2}+\phi_{2 i, 2 j-1}^{h / 2}+\phi_{2 i-1,2 j-1}^{h / 2}\right)
$$

and correspondingly for $e^{2 h: h}$. The rate of convergence is defined as

$$
\log _{2}\left(\frac{\left\|e^{2 h: h}\right\|_{2}}{\left\|e^{h: h / 2}\right\|_{2}}\right)
$$

The errors and rate of convergence are shown in Table (4.1) which indicates that the scheme is of second-order in both space and time. Furthermore, we plot in Fig. 4.1 (left) the interface contour with different time steps to show that the numerical solution quickly converges as we decrease the time step.

TABLE 4.1. Errors and convergence rate for the scheme (3.16a)-(3.16e).

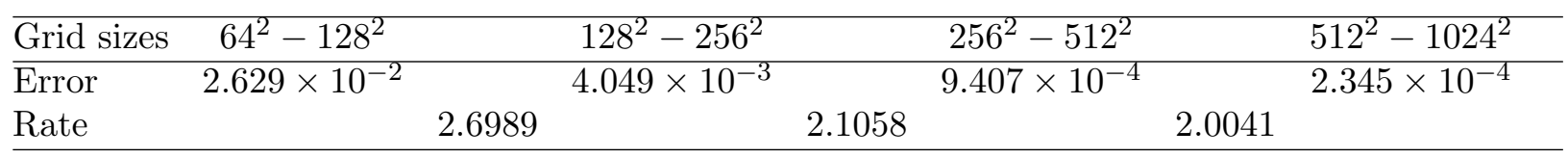

Next, we examine the savings achieved by the space and time adaptivity. In Table 4.2 , we list the CPU time consumed by using the constant time step/uniform meshes, constant time step/adaptive meshes, and adaptive time step/adaptive meshes with two different set of parameters. It is observed that the computational efficiency is significantly improved by using adaptive time steps and adaptive meshes. Because the scheme is unconditionally stable, it is more efficient to use a large upper bound $\Delta t_{\text {max }}$. In Fig. 4.1 (right), a typical mesh is shown. We observe that the mesh is properly refined near the interfacial region. 

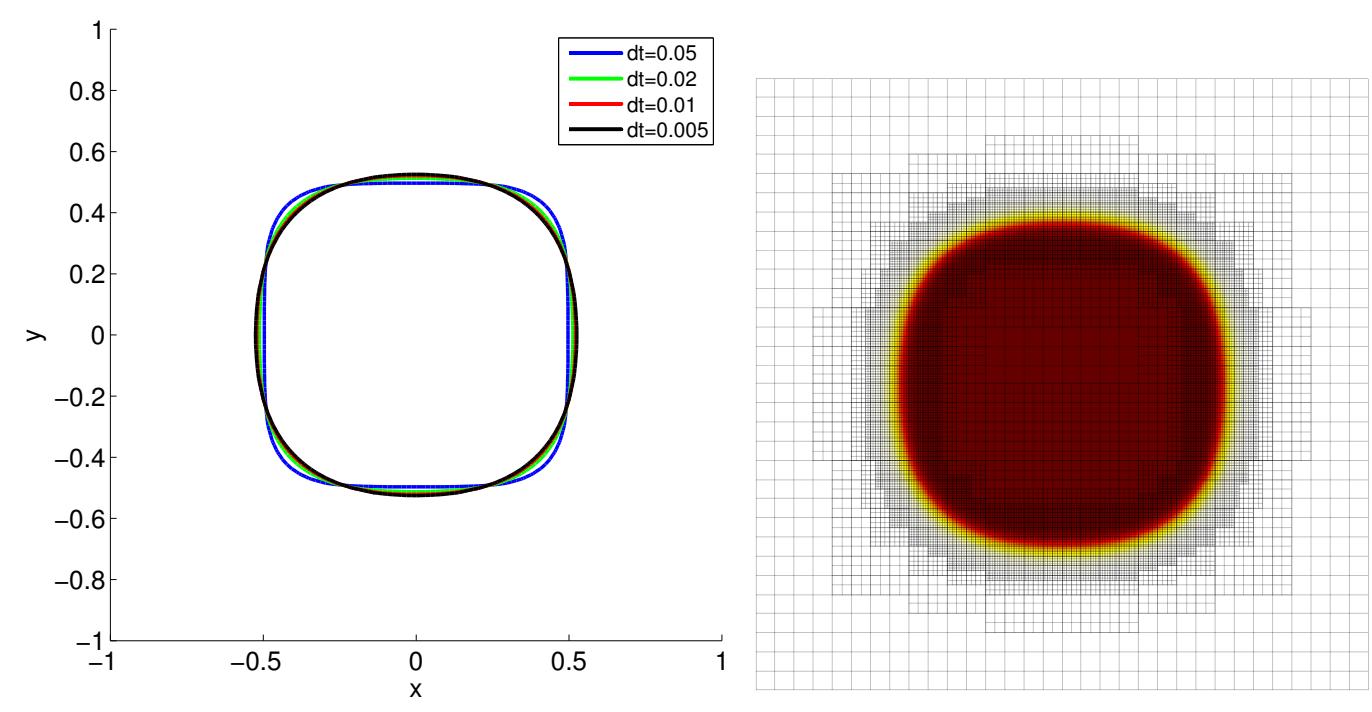

FiguRE 4.1. Left: the $\phi=0$ contours at $t=2.0$ using different time steps and uniform meshes; Right: a typical adaptive mesh. Three levels of refinement are performed with the root-level grid $32 \times 32$.

TABle 4.2. CPU time (in seconds) comparison for Example 1. The constant time step is set at $\Delta t=0.01$. We choose in (3.15) $\Delta t_{\text {min }}=0.01, \alpha=10000$ with two different choices of $\Delta t_{\max }$ : Results with $\Delta t_{1}$ os obtained with $\Delta t_{\max }=0.1$, while results with $\Delta t_{2}$ os obtained with $\Delta t_{\max }=1$.

\begin{tabular}{|c|r|r|r|r|r|r|r|r|r|r|r|}
\hline$t$ & 0.2 & 0.5 & 1 & 2 & 4 & 20 & 100 & 500 & 1000 & 2000 & 3000 \\
\hline $\begin{array}{c}\text { const. } \Delta t \\
\text { uni. meshes }\end{array}$ & 6.5 & 13.2 & 24.2 & 45.4 & 83.9 & 324.3 & 1092.3 & 6425.2 & 13744.0 & 23455.0 & 31788.0 \\
\hline $\begin{array}{c}\text { const. } \Delta t \\
\text { ad. meshes }\end{array}$ & 3.4 & 6.7 & 11.6 & 24.4 & 46.2 & 204.5 & 891.1 & 4380.3 & 8429.7 & 13256.0 & 18074.0 \\
\hline $\begin{array}{c}\text { ad. } \Delta t_{1} \\
\text { ad. meshes }\end{array}$ & 3.4 & 6.6 & 11.6 & 21.7 & 28.1 & 59.0 & 131.0 & 455.3 & 892.9 & 1860.4 & 2667.3 \\
\hline $\begin{array}{c}\text { ad. } \Delta t_{2} \\
\text { ad. meshes }\end{array}$ & 3.5 & 6.0 & 8.6 & 10.5 & 12.9 & 31.4 & 54.1 & 157.6 & 273.6 & 501.4 & 777.6 \\
\hline
\end{tabular}

4.2. Example 2: Coarsening dynamics. We take the same parameters as in the first example but start with a random phase function in the domain $[-1,1] \times[-1,1]$ to simulate the coarsening dynamics in the presence of flow.

Characteristic evolutions of the phase functions at different times are shown in Fig. 4.5. In the right of Fig. 4.2, we plot the total energy evolution which decreases as the system evolves. CPU time consumed by the constant time step/uniform meshes, constant time step/adaptive meshes, and adaptive time step/adaptive meshes is shown in Table 4.3. We observe that the fully adaptive scheme is still much more efficient than their non-adaptive counterpart, but compared to Example 1 , the CPU saying is less impressive due to the larger percentage of interfacial region in this case.

4.3. Example 3: Lighter bubble rising in a heavier medium. In order to demonstrate that our fully adaptive scheme is robust with respect to density variations, we consider the situation 

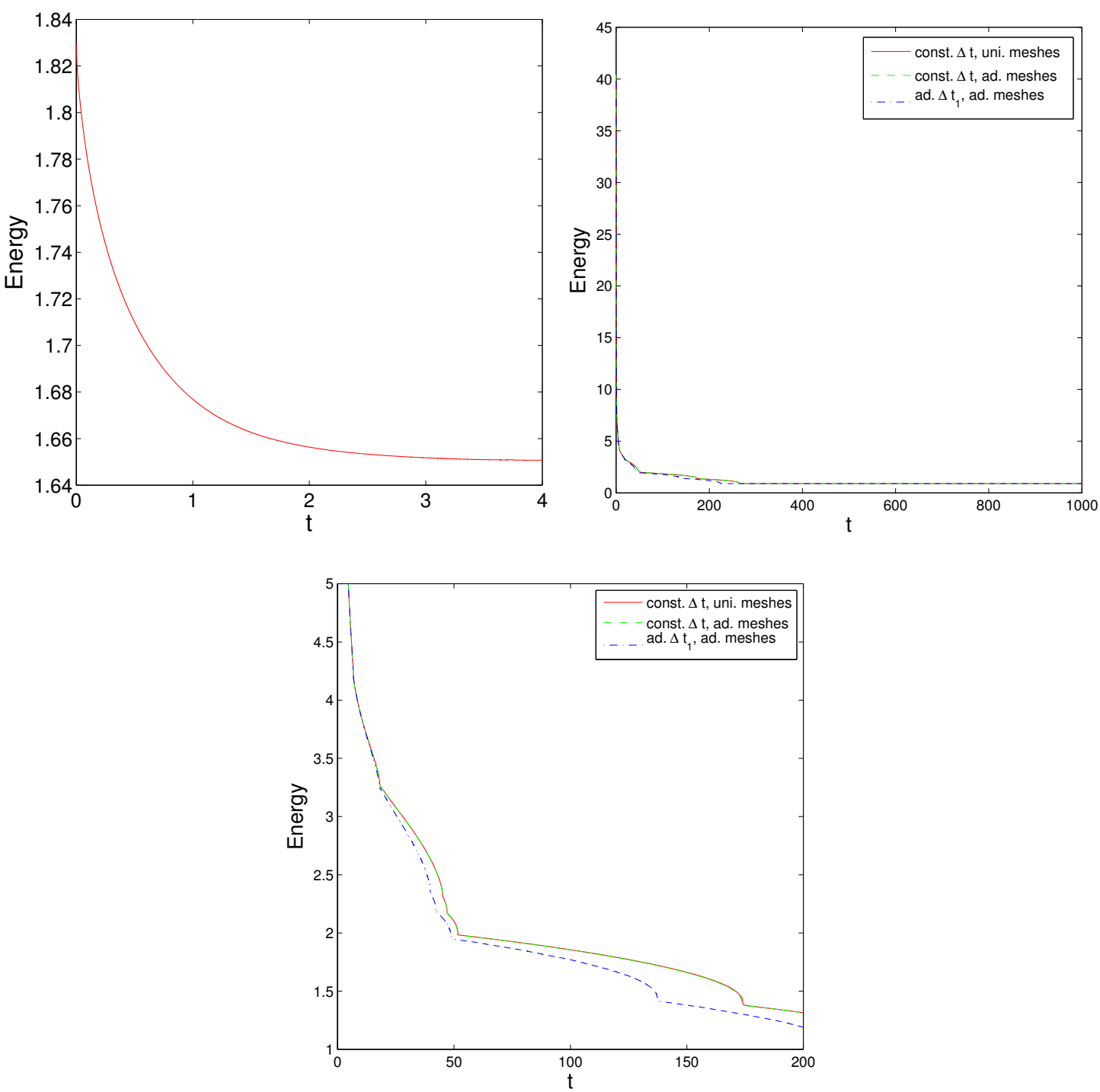

Figure 4.2. The evolution of discrete energy for Example 1 (Top/left) and Example 2 (Top/right) using the second-order scheme. The bottom graph is a part of figure showing the evolution of the discrete energy for Example 2.

TABle 4.3. CPU time (seconds) comparison for Example 2. The constant time step is set at $\Delta t=0.01$. We choose in (3.15) $\Delta t_{\min }=0.01, \Delta t_{\min }=1, \alpha=10000$.

\begin{tabular}{|c|c|r|r|r|r|r|r|r|r|r|r|}
\hline$t$ & 0.2 & 0.5 & 1 & 2 & 4 & 20 & 100 & 500 & 1000 & 2000 & 3000 \\
\hline $\begin{array}{c}\text { const. } \Delta t \\
\text { uni. meshes }\end{array}$ & 6.9 & 14.4 & 28.9 & 52.3 & 98.7 & 434.4 & 2152.9 & 8496.1 & 12396.0 & 20050.0 & 27545.0 \\
\hline $\begin{array}{c}\text { const. } \Delta t \\
\text { ad. meshes }\end{array}$ & 8.1 & 16.4 & 30.7 & 59.0 & 111.5 & 476.1 & 1674.3 & 3613.1 & 4503.2 & 6165.3 & 7850.1 \\
\hline $\begin{array}{c}\text { ad. } \Delta t_{1} \\
\text { ad. meshes }\end{array}$ & 7.6 & 15.9 & 31.5 & 57.0 & 109.4 & 441.6 & 1463.4 & 2376.4 & 2509.5 & 2700.3 & 2889.2 \\
\hline
\end{tabular}


where a lighter bubble (with density $\rho_{1}$ and dynamic viscosity $\nu_{1}$ ) initially inside a heavier medium (with density $\rho_{2}$ and dynamic viscosity $\left.\nu_{2}\right)$ confined in a rectangular domain $\Omega=(0, \mathrm{~d}) \times\left(0, \frac{3}{2} \mathrm{~d}\right.$ ).

The equations are non-dimensionalized using the following scaled variables:

$$
\tilde{t}=\frac{t}{t_{0}}, \tilde{\rho}=\frac{\rho}{\rho_{0}}, \tilde{x}=\frac{x}{d_{0}}, \tilde{u}=\frac{u}{u_{0}},
$$

where

$$
t_{0}=\sqrt{d / g}, d_{0}=d ; u_{0}=\sqrt{d g}, \rho_{0}=\min \left(\rho_{1}, \rho_{2}\right) .
$$

The dimensionless form of (2.7) with an extra gravitational force $\rho g$ in the momentum equation, after omitting the $\sim$ from the notation, is:

$$
\begin{aligned}
& \phi_{t}+M \Delta \mu+\nabla \cdot(\phi \mathbf{u})=0 \\
& \mu+\lambda\left(\frac{\phi\left(\phi^{2}-1\right)}{\eta^{2}}-\Delta \phi\right)=0 \\
& \left.\rho\left(\mathbf{u}_{t}+(\mathbf{u} \cdot \nabla) \mathbf{u}\right)\right)+\mathbf{J} \cdot \nabla \mathbf{u}-\nabla \cdot(\nu \nabla \mathbf{u})+\nabla p-\phi \Delta \mu=-\rho g, \\
& \nabla \cdot \mathbf{u}=0
\end{aligned}
$$

with

$$
\mathbf{J}=-\frac{\tilde{\rho}_{1}-\tilde{\rho}_{2}}{2} M \nabla \mu, \rho(\phi)=\frac{\tilde{\rho}_{1}-\tilde{\rho}_{2}}{2} \phi+\frac{\tilde{\rho}_{1}+\tilde{\rho}_{2}}{2}, \nu(\phi)=\frac{\tilde{\nu}_{1}-\tilde{\nu}_{2}}{2} \phi+\frac{\tilde{\nu}_{1}+\tilde{\nu}_{2}}{2} .
$$

where $\tilde{\rho}_{1}=\rho_{1} / \rho_{0}, \tilde{\rho}_{2}=\rho_{2} / \rho_{0}, \tilde{\nu}_{1}=\nu_{1} /\left(\rho_{0} \mathrm{~d}^{3 / 2} \mathrm{~g}^{1 / 2}\right), \tilde{\nu}_{2}=\nu_{2} /\left(\rho_{0} \mathrm{~d}^{3 / 2} \mathrm{~g}^{1 / 2}\right)$.

The initial velocity and pressure are set to be zero and initial phase function is given by

$$
\phi(x, t=0)=-\tanh \left(\frac{\mathrm{r}-\frac{1}{4} \mathrm{~d}}{\sqrt{2} \eta_{0}}\right),
$$

where $\mathrm{r}$ is the distance from the center of the bubble to the point and $\eta_{0}$ is the diffusive interfacial width.

we set $d=1, g=(0,9.8)^{T}, M=0.002, \nu=1, \lambda=0.05$, and $\eta_{0}=\eta=0.02$, and test two cases with density ratios $1: 10,1,1: 40$, respectively. The initial time step size is $\Delta t=0.0002$. We use the root-level grid size $128 \times 192$ and perform three levels of mesh refinement. Snapshots of phase evolution are shown in Fig. 4.6 for the density ratio (1:10) and in Fig. 4.7 for the density ratio (1:40). We plot in Fig. 4.8 a typical mesh refinement for this example. Once again, we observe that the mesh is properly refined near the interfacial region.
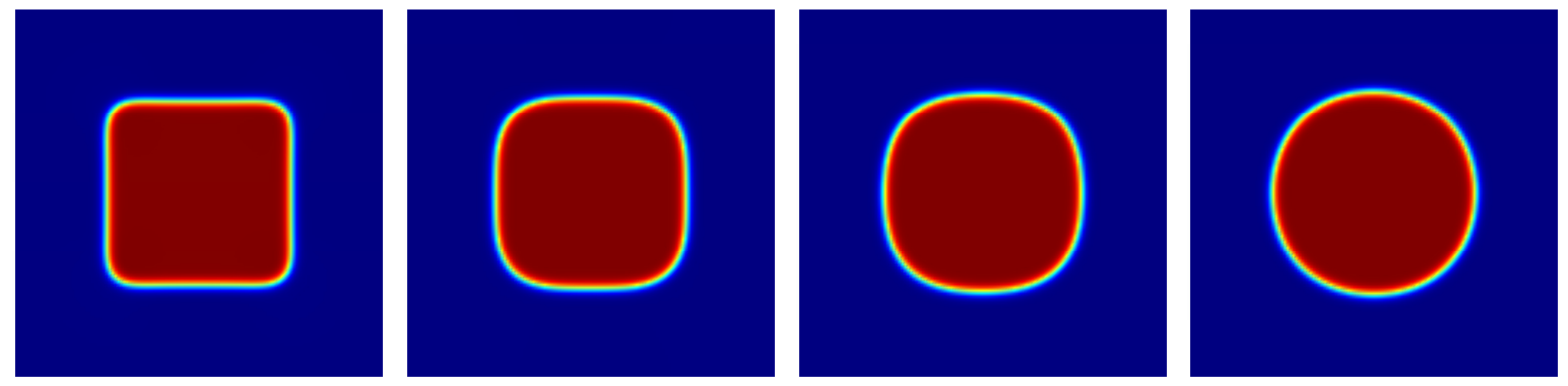

Figure 4.3. Snapshots of the phase function in Example 1 at $T=0.2,1,2,4$, respectively. 

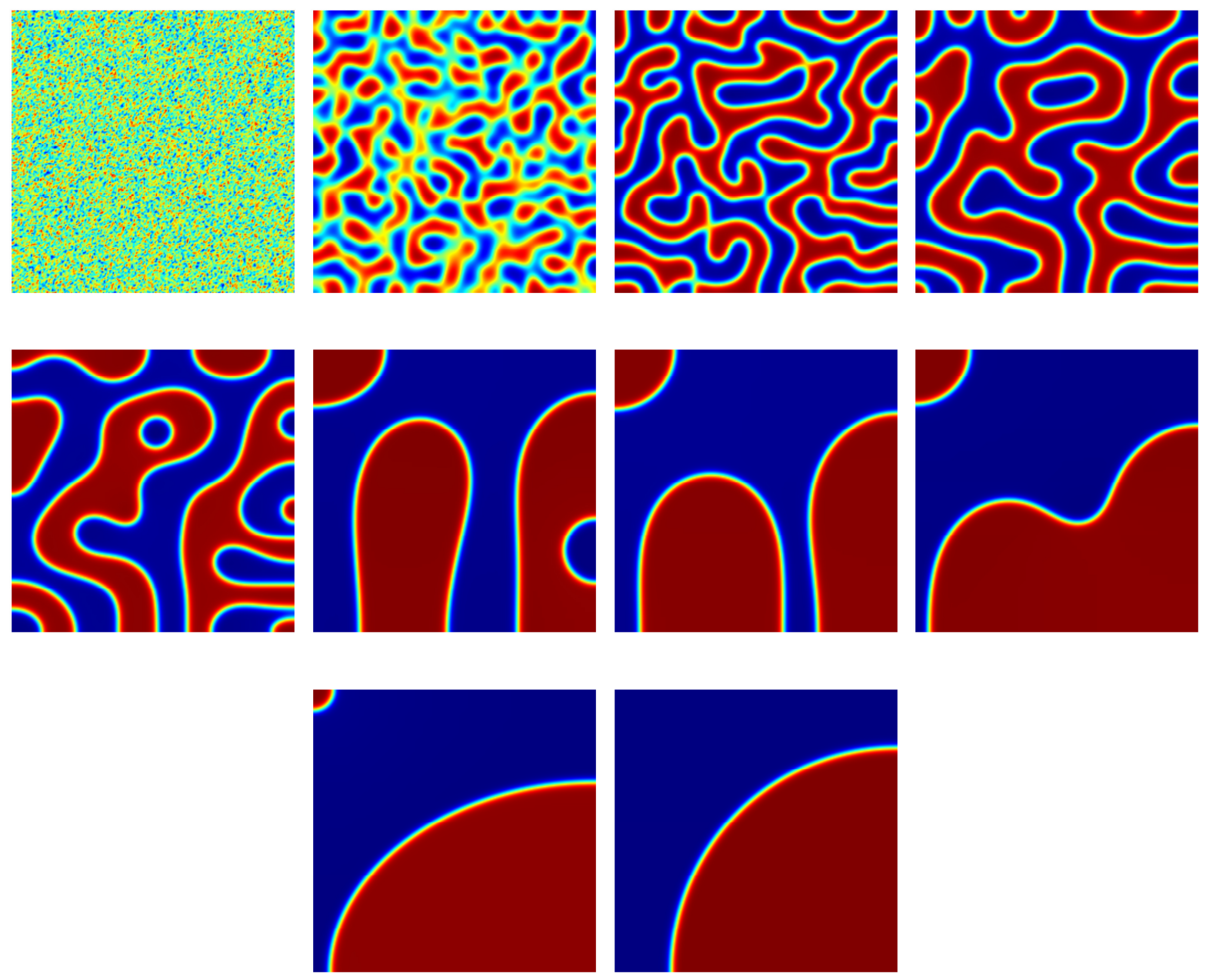

Figure 4.4. Snapshots of the phase function in Example 2 at $T=0,0.2,0.5,1,2$, $20,50,70,100,500$, respectively, using adaptive meshes with the root-level grid size $32 \times 32$ and two levels of refinement, and adaptive time with $\Delta t_{\max }=1$.

4.4. Summary and future work. We developed space and time adaptive numerical methods in this paper for the Cahn-Hilliard Navier-Stokes phase-field models for incompressible two phase flows with uniform and variable densities. We adopted the decoupled, linear, and energy stable schemes constructed in [28] and coupled them with an adaptive mesh refinement with finite-differences and an adaptive time approach. We showed rigorously that our fully discrete scheme is also unconditionally energy stable.

This fully adaptive scheme is extremely efficient, as it only requires solving a sequence of decoupled linear equations at each time step, and it adjusts the time step and spatial resolution automatically. We presented several illustrative numerical results to show that our fully adaptive scheme can save CPU and memory requirement significantly for this type of interface problems.

While we have only provided numerical results for two-dimensional simulations, the method extends naturally to three-dimensional cases. We are also extending the approaches developed in this paper to study more complex two-phase flows such as tumor growth model [20].

Acknowledgment. This work is partially supported by NSF DMS-1217066 and DMS-1419053. 

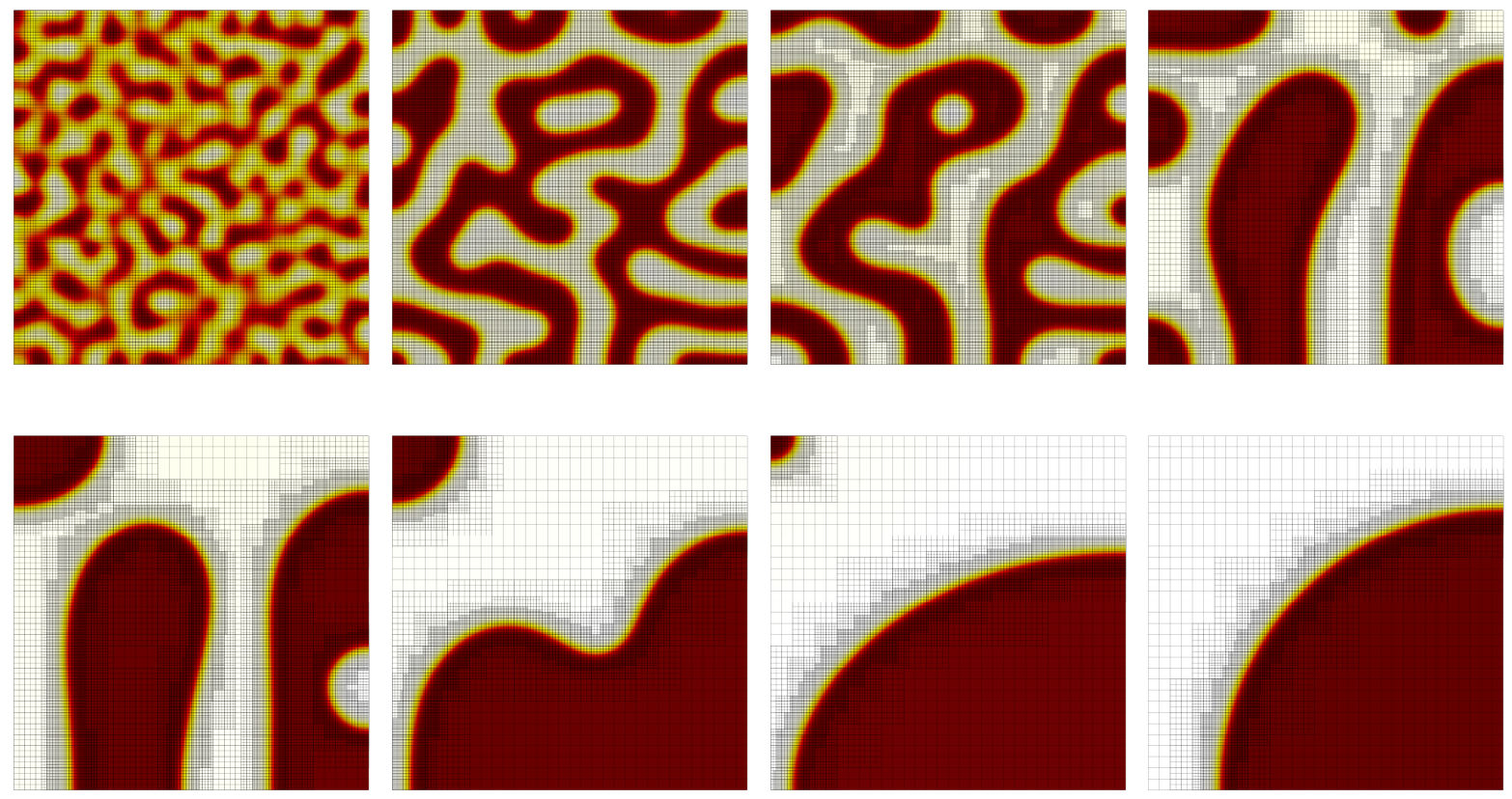

FigurE 4.5. Snapshots of the phase function in Example 2. Three levels of refinement are performed: the root-level grid is $32 \times 32$.

\section{REFERENCES}

[1] H. Abels. Existence of weak solutions for a diffuse interface model for viscous, incompressible fluids with general densities. Comm. Math. Phys., 289(1):45-73, 2009.

[2] H. Abels, H. Garcke, and Gü. Grün. Thermodynamically consistent, frame indifferent diffuse interface models for incompressible two-phase flows with different densities. Mathematical Models and Methods in Applied Sciences, $22(03), 2012$.

[3] D. M. Anderson, G. B. McFadden, and A. A. Wheeler. Diffuse-interface methods in fluid mechanics. 30:139-165, 1998.

[4] R. Becker, X. Feng, and A. Prohl. Finite element approximations of the Ericksen-Leslie model for nematic liquid crystal flow. SIAM J. Numer. Anal., 46(4):1704-1731, 2008.

[5] F. Boyer. Nonhomogeneous Cahn-Hilliard fluids. Ann. Inst. H. Poincaré Anal. Non Linéaire, 18(2):225-259, 2001.

[6] J. W. Cahn and J. E. Hilliard. Free energy of a nonuniform system. I. interfacial free energy. J. Chem. Phys., 28:258-267, 1958.

[7] P. M. Chaikin and T.C. Lubensky. Principles of Condensed Matter Physics. Cambridge, 1995.

[8] Y. Chen, S. Wise, V. Shenoy, and J. Lowengrub. A stable scheme for a nonlinear, multispecies tumor growth model with an elastic membrane. Int. J. Numer. Meth. Biomed. Engng., 30:726-754, 2014.

[9] C. Collins, J. Shen, and S. Wise. An efficient, energy stable scheme for the cahn-hilliard-brinkman system. Commun. Comput. Phys., 13:929-957, 2013.

[10] H. Ding, P. D. M. Spelt, and C. Shu. Calculation of two-phase Navier-Stokes flows using phase-field modeling. J. Comput. Phys., 226(2):2078-2095, 2007.

[11] X. Feng, Y. He, and C. Liu. Analysis of finite element approximations of a phase field model for two-phase fluids. Math. Comp., 76(258):539-571 (electronic), 2007.

[12] M. E. Gurtin, D. Polignone, and J. Viñals. Two-phase binary fluids and immiscible fluids described by an order parameter. Math. Models Methods Appl. Sci., 6(6):815-831, 1996.

[13] D. Han and X. Wang. A second order in time, uniquely solvable, unconditionally stable numerical scheme for cahn-hilliard-navier-stokes equation. J. Comput. Phys., 290:139-156, 2015.

[14] F.H. Harlow and J.E. Welch. Numerical calculation of time-dependent viscous incompressible flow of fluid with free surface. Phys. Fluids, 8:2182-2189, 1965. 

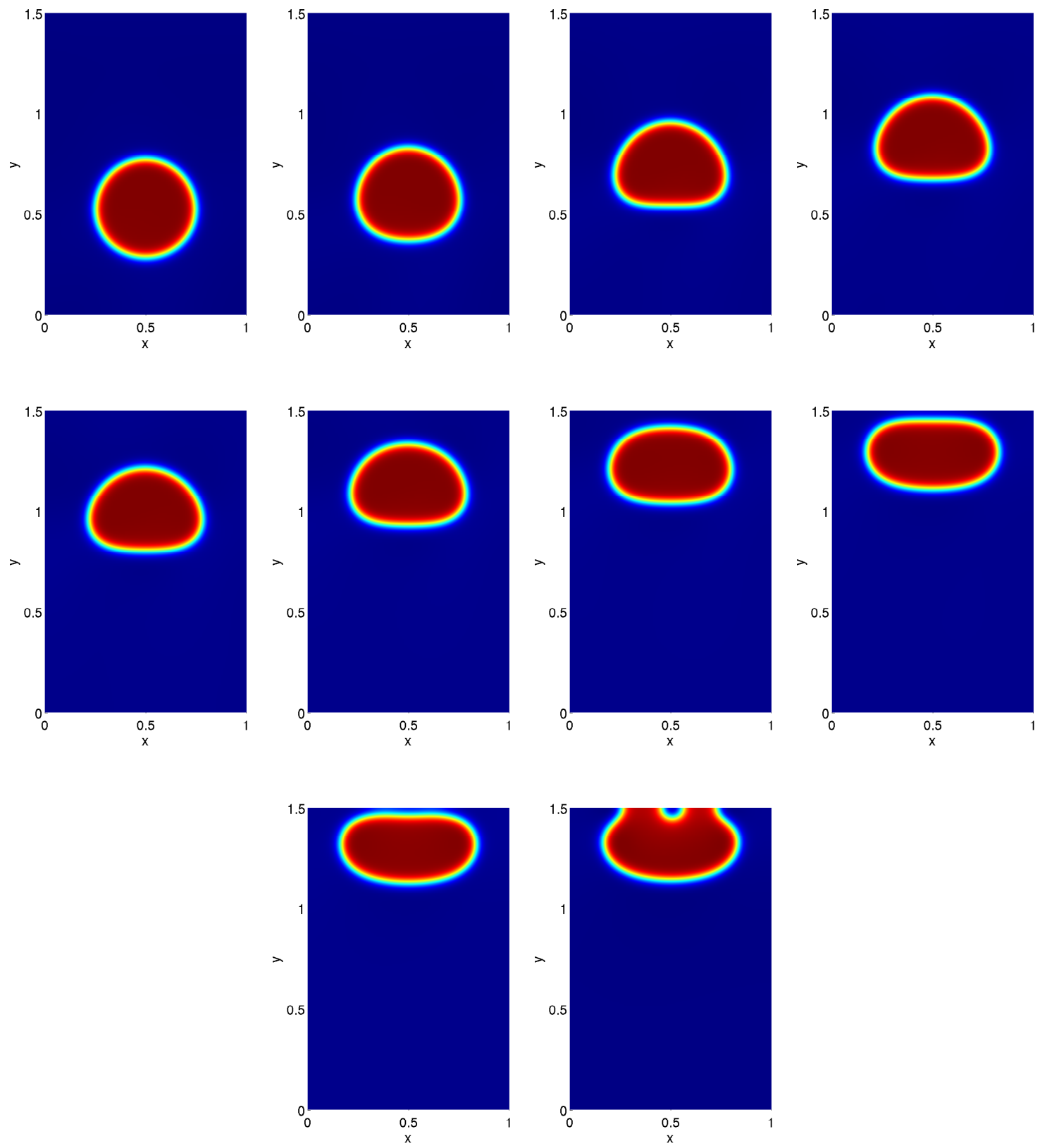

Figure 4.6. The evolution of a rising drop with density ratio $1: 10$ at $T=$ $0.125,0.25,0.5,0.75,1.0,1.25,1.5,1.75,1.875,2.0$, respectively.

[15] D. Jacqmin. Diffuse interface model for incompressible two-phase flows with large density ratios. J. Comput. Phys., 155(1):96-127, 2007.

[16] J. Kim. Phase-field models for multi-component fluid flows. Commun. Comput. Phys., 12(3):613-661, 2012.

[17] P. Lin, C. Liu, and H. Zhang. An energy law preserving $C^{0}$ finite element scheme for simulating the kinematic effects in liquid crystal dynamics. J. Comput. Phys., 227(2):1411-1427, 2007.

[18] C. Liu and J. Shen. A phase field model for the mixture of two incompressible fluids and its approximation by a Fourier-spectral method. Physica D, 179(3-4):211-228, 2003. 

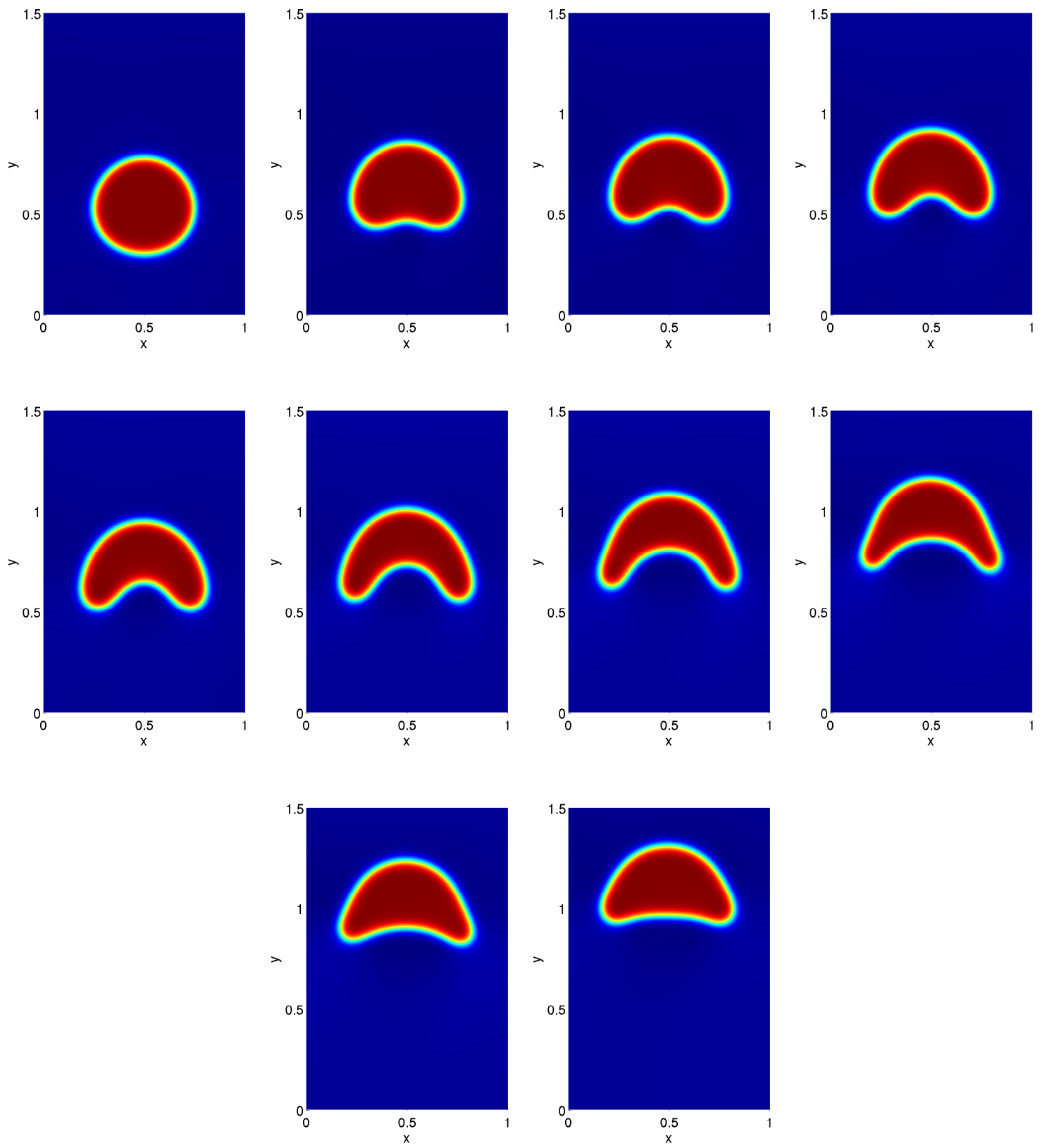

Figure 4.7. The evolution of a rising drop with density ratio $1: 40$ at $T=$ $0.125,0.25,0.3,0.35,0.4,0.5,0.625,0.75,0.875,1.0$, respectively.

[19] C. Liu, J. Shen, and X. Yang. Decoupled energy stable schemes for a phase-field model of two-phase incompressible flow with variable density. J. Sci. Comput, DOI 10.1007/s10915-014-9867-4, 2014.

[20] J. Lowengrub, E. Titi, and K. Zhao. Analysis of a mixture model of tumor growth. Europ. J. Appl. Math., 24:691-734, 2013.

[21] J. Lowengrub and L. Truskinovsky. Quasi-incompressible Cahn-Hilliard fluids and topological transitions. Proc. R. Soc. Lond. A Math. Phys. Eng. Sci., 454:2617-2654, 1998.

[22] M. L. Minion. A projection method for locally refined grids. J. Comput. Phys., 127:158-178, 1996. 


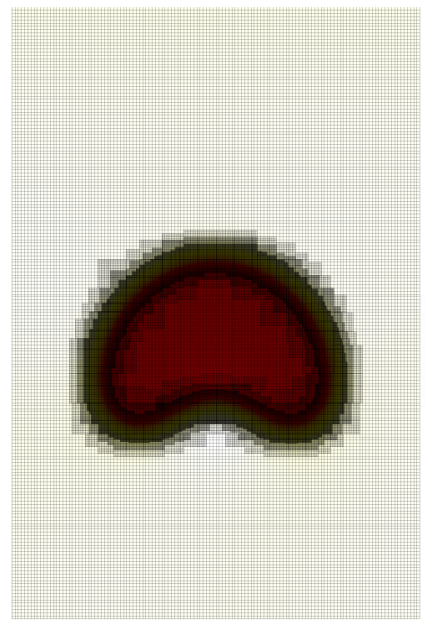

Figure 4.8. Example 3: a typical adaptive mesh. Three levels of refinement are performed: the root-level grid is $128 \times 196$.

[23] Z.H. Qiao, Z.R. Zhang, and T. Tang. An adaptive time-stepping strategy for the molecular beam epitaxy models. SIAM J. Sci. Comput., 33:1395-1414, 2011.

[24] L. Rayleigh. On the theory of surface forces ii. Phil. Mag., 33:209, 1892.

[25] J. Shen. Modeling and numerical approximation of two-phase incompressible flows by a phase-field approach. In Multiscale Modeling and Analysis for Materials Simulation, Lecture Note Series, Vol. 9. IMS, National University of Singapore, 2011, Edited by W. Bao and Q. Du, pages 147-196. 2011.

[26] J. Shen, Q. Wang, and X. Yang. Mass-conserved phase field models for binary fluids. Comm. Comput. Phys., 13:1045-1065, 2013

[27] J. Shen and X. Yang. Energy stable schemes for cahn-hilliard phase-field model of two-phase incompressible flows. Chinese Ann. Math. series B, 31:743-758, 2010.

[28] J. Shen and X. Yang. Decoupled, energy stable schemes for phase-field models of two-phase incompressible flows. submitted, 2013.

[29] M. Sussman, A.S. ALmgren, J.B. Bell, P. Colella, and L.H. Howell. An adaptive level set approach for incompressible two-phase flows. J. Comput. Phys., 148:81-124, 1999.

[30] J. van der Waals. The thermodynamic theory of capillarity under the hypothesis of a continuous density variation. J. Stat. Phys., 20:197-244, 1893.

[31] S. Wise. Unconditionally stable finite difference, nonlinear multigrid simulation of the cahn-hilliard-hele-shaw system of equations. J. Sci. Comput., 44:38-68, 2010.

[32] S. Wise, J. Lowengrub, and V. Cristini. An adaptive multigrid algorithm for simulating solid tumor growth using mixture models. Math. Comput. Model., 53:1-20, 2011.

[33] S. Wise, C. Wang, and J. Lowengrub. An energy stable and convergent finite-difference scheme for the phase field crystal equation. SIAM J. Numer. Anal., 47:2269-2288, 2009.

[34] S.M. Wise, J. Kim, and J.S. Lowengrub. Solving the regularized, strongly anisotropic cahn-hilliard equation by an adaptive nonlinear multigrid method. J. Comput. Phys., 226:414-446, 2007.

[35] S.M. Wise, J.S. Lowengrub, H.B. Frieboes, and V. Cristini. Three-dimensional multi species nonlinear timor growth-i model and numerical method. J. Theor. Biol., 253:524-543, 2008.

[36] P. Yue, J. J. Feng, C. Liu, and J. Shen. A diffuse-interface method for simulating two-phase flows of complex fluids. J. Fluid Mech, 515:293-317, 2004.

[37] Z.R. Zhang, Y. Ma, and Z.H. Qiao. An adaptive time-stepping strategy for solving the phase field crystal model. J. Comput. Phys., 249:204-215, 2013. 